\title{
Formation initiale et modèles d'enseignement de nouveaux enseignants au collégial
}

Diane Leduc, Louise Ménard et Éric Le Coguiec

\section{(2) OpenEdition}

Édition électronique

URL : http://journals.openedition.org/ripes/855

ISSN : 2076-8427

Éditeur

Association internationale de pédagogie universitaire

Référence électronique

Diane Leduc, Louise Ménard et Éric Le Coguiec, « Formation initiale et modèles d'enseignement de nouveaux enseignants au collégial », Revue internationale de pédagogie de l'enseignement supérieur [En ligne], 30(3) | 2014, mis en ligne le 01 décembre 2014, consulté le 08 septembre 2020. URL : http:// journals.openedition.org/ripes/855

Ce document a été généré automatiquement le 8 septembre 2020

Article L.111-1 du Code de la propriété intellectuelle. 


\title{
Formation initiale et modèles d'enseignement de nouveaux enseignants au collégial
}

\author{
Diane Leduc, Louise Ménard et Éric Le Coguiec
}

\section{Introduction}

1 Au Québec, aux États-Unis et dans certains pays d'Europe, ceux qui veulent enseigner au postsecondaire n'ont pas l'obligation d'acquérir une formation en enseignement. Le diplôme supérieur spécialisé dans une discipline est généralement exigé pour obtenir une charge d'enseignement dans un collège. Pourtant, les transformations des apprenants, caractérisées notamment par la diversité de leur itinéraire scolaire et l'importance relative qu'ils accordent aux études, témoignent de nouvelles réalités auxquelles les enseignants doivent faire face (CSE, 2000). Cette situation incite les administrations de plusieurs collèges, qui cherchent aussi à augmenter le taux de persévérance et de réussite de leurs étudiants, à se questionner sur la qualité de l'enseignement et sur leur capacité à mieux les encadrer. C'est la raison pour laquelle elles valorisent et offrent, depuis plusieurs années, de la formation en enseignement, créditée et non créditée, aux enseignants qui souhaitent se former ou se perfectionner. Pour répondre à cette demande de formation, l'Université du Québec à Montréal (UQÀM) a créé, dès 1998 , le programme court de $2^{\mathrm{e}}$ cycle en pédagogie de l'enseignement supérieur (PCPES).

2 C'est pour améliorer la qualité de ce programme d'études, ainsi que pour répondre aux besoins de nouveaux enseignants et de collèges, que nous avons sondé des diplômés du programme qui ont obtenu une charge d'enseignement dans un collège. Nous souhaitions savoir s'ils utilisent les modèles d'enseignement appris au cours de leur formation initiale. Cette modeste étude, effectuée à l'aide d'un questionnaire en ligne, représente la première phase de travaux plus larges qui visent à mieux arrimer la 
formation initiale aux réalités en salle de classe et aux contingences actuelles qui bousculent le milieu de l'enseignement au postsecondaire.

Dans cet article, une fois la problématique exposée, nous contextualiserons et décrirons la formation initiale (le PCPES) de laquelle sont issus les sujets à l'étude et nous préciserons les modèles d'enseignement qui y sont enseignées en mettant en relief la manière dont ils sont abordés dans la formation. Puis, nous décrirons la méthodologie et le déroulement de l'étude avant de discuter des résultats.

\section{Un enseignement au service des apprentissages : la révision des modèles pédagogiques}

4 Hormis dans quelques disciplines comme l'éducation et certains programmes en santé et en sciences et malgré l'implantation d'approches par compétences, plusieurs pratiques enseignantes au collégial sont encore orientées sur le paradigme centré sur l'enseignement où la formation est considérée comme une transmission de savoirs et où l'enseignant est l'expert et principal responsable de cette transmission (Langevin, 2007 ; St-Pierre, Bédard \& Lefebvre, 2012). Or, selon Romainville (2004) et Cullen, Richardson et O'Brien (2004), la transmission linéaire du savoir s'avère de plus en plus lacunaire. On reproche surtout aux méthodes traditionnelles d'être limitées à la transmission des connaissances vers des étudiants passifs et peu motivés (Bertrand, 1998; Brauer, 2011). À l'opposé, plusieurs réformes éducatives s'appuient sur des théories actuelles et visent à appliquer les principes du paradigme centré sur l'apprentissage. Sous cette égide, la formation est vue comme un dispositif mis en oeuvre pour faciliter les apprentissages des apprenants. Il prend en compte leur motivation et leurs stratégies d'apprentissage en articulation avec les actions de l'enseignant. Il semble que les modèles d'enseignement dits traditionnels, très centrés sur l'enseignant, laissent graduellement la place à des modèles dits actifs, plus centrés sur l'étudiant, capable de construire son savoir et ses apprentissages (Meirieu, 2009).

5 Les valeurs véhiculées au PCPES souscrivent à cette dernière vision. Cependant, il est aussi bien connu que les enseignants (comme les étudiants) sont profondément imprégnés du modèle d'enseignement auquel ils ont été confrontés durant toutes leurs années d'études (Loiola \& Tardif, 2001). En début de carrière, plusieurs tendent à enseigner en imitant les façons de faire des enseignants qu'ils ont eus à titre d'étudiants. Considérant les difficultés que vit un nouvel enseignant dans un collège, c'est une stratégie rassurante et utile pour favoriser son intégration professionnelle. N'ayant peu ou pas enseigné, la plupart des étudiants du PCPES n'échappent pas à ce constat. C'est l'un des principaux buts de cette formation que d'agir sur les types d'enseignement reçus en faisant vivre aux étudiants une variété de modèles. La recherche tend d'ailleurs à démontrer qu'il est préférable pour un enseignant de ne pas se limiter à un seul modèle d'enseignement (Brauer, 2011; De Koninck, 1996; Raby \& Viola, 2007). L'utilisation de plusieurs modèles serait par conséquent à privilégier. Ramsden (2003) rapporte aussi que les modèles d'enseignement des professeurs ont un impact sur les apprentissages réalisés par les étudiants et qu'ils ne peuvent être dissociés des contextes, que sont notamment la classe et l'établissement dans lesquels ils prennent place.

6 Mais, dans certaines situations, le contexte n'est pas toujours favorable au réinvestissement des contenus de la formation initiale, dont font partie les modèles 
d'enseignement, tel qu'ils ont été enseignés. En effet, lorsqu'un enseignant amorce sa carrière dans un établissement d'enseignement, divers éléments propres au milieu d'accueil facilitent ou rendent difficile l'utilisation de modèles d'enseignement variés. Pour de multiples raisons, les jeunes enseignants, davantage centrés sur eux-mêmes et sur le contenu des cours (Feyfant, 2011; Langevin, 2007), ne peuvent pas toujours appliquer les notions apprises durant leur formation dans leur pratique quotidienne. Pourtant, selon Ménard (2009), la formation avant l'embauche a plus d'impacts que celle offerte lorsque les enseignants sont intégrés dans le milieu. Le climat de travail, la gestion du temps, les relations interpersonnelles avec les étudiants et les collègues, la précarité, l'expérience en enseignement, sont autant d'aspects auxquels les nouveaux enseignants sont confrontés dès leur entrée en poste. Ainsi, malgré le développement des pratiques pédagogiques au collégial et malgré le haut niveau de compétences exigées à l'embauche des enseignants, plusieurs circonstances les empêchent de réinvestir certains apprentissages réalisés durant la formation initiale (Héon, Savard \& Hamel, 2008 ; St-Pierre, 2007).

7 Nous prenons donc comme prémisse que plusieurs conditions, telles que la formation initiale reçue, influencent l'application en salle de classe des modèles d'enseignement. Dans l'optique de vérifier la pertinence des contenus enseignés au PCPES, nous nous sommes posés les questions suivantes: les diplômés du PCPES utilisent-ils les divers modèles d'enseignement appris? Si oui, qu'est-ce qui facilite l'utilisation de ces modèles? Si non, qu'est-ce qui les restreint? Le lecteur notera que notre étude se limite à un seul aspect de la formation initiale, soit les modèles d'enseignement.

\section{L'offre de formations initiales pour l'enseignement au collégial}

8 Pour pouvoir enseigner au Québec au niveau collégial, il faut détenir au minimum un baccalauréat spécialisé de premier cycle (généralement d'une durée de trois ans) correspondant à une discipline enseignée au collégial et parfois une maîtrise (exigée notamment pour enseigner la littérature, l'histoire, la philosophie). Dans certains cas, le diplôme de doctorat est même exigé, notamment pour enseigner la psychologie et souvent demandé pour enseigner les mathématiques, la chimie, la physique ou la biologie (Héon, Savard \& Hamel, 2008). Afin de répondre aux demandes des collèges québécois, publics ou privés, en matière de formation initiale en enseignement supérieur, les établissements collégiaux et universitaires se sont dotés de programmes de formation initiale en pédagogie de l'enseignement supérieur. Six universités québécoises (les universités du Québec à Montréal et à Chicoutimi ainsi que les universités Laval, de Montréal, de Sherbrooke et McGill) se partagent plus de huit de ces formations. Après examen des cours offerts dans ces différentes formations, nous pouvons dégager comme visées communes qu'un enseignant du collégial devrait non seulement être expert de sa discipline, mais aussi être capable de transmettre un savoir plus ou moins spécialisé, avoir une compréhension minimale des modèles d'apprentissage, des caractéristiques des étudiants ainsi que de la pédagogie et avoir une habileté marquée à travailler en équipe. Par ailleurs, plusieurs des formations disponibles actuellement offrent des cours sur les modèles d'enseignement. Il faut aussi noter qu'au Québec, certaines formations mentionnées précédemment s'adressent aux 
aspirants enseignants alors que d'autres s'offrent aux enseignants déjà embauchés (StPierre, 2007).

\subsection{Le programme court de $2^{\mathrm{e}}$ cycle en pédagogie de l'enseignement supérieur (PCPES)}

Le Programme court de $2^{\mathrm{e}}$ cycle en pédagogie de l'enseignement supérieur (PCPES) a accueilli sa première cohorte d'étudiants à l'automne 1998. Sans égards aux disciplines, le PCPES est conçu avant tout pour les futurs enseignants et chargés de cours des collèges. Il vise à développer les habiletés de base pour enseigner au niveau postsecondaire en tenant compte du contexte institutionnel, des besoins des étudiants, de l'intégration en classe des technologies ainsi que des principes et des méthodes caractéristiques d'un enseignement efficace. Le programme est basé sur des valeurs, des compétences et des contenus qui suivent différents modèles ancrés dans une approche socioconstructiviste de l'enseignement et il tient compte de la réforme, basée sur une approche-programme par compétences, mise en place dans les collèges québécois à partir de 1994.

10 Bien que le programme s'adresse à l'enseignement supérieur, la majorité des 200 étudiants actifs du PCPES souhaitent enseigner au niveau collégial et les cours sont adaptés en conséquence. Pour recevoir leur attestation de $2^{\mathrm{e}}$ cycle, les personnes inscrites doivent suivre et réussir cinq cours de 45 heures chacun (ou quatre cours et un stage). Les compétences à atteindre concernent la planification de cours et de séances, l'adaptation d'un enseignement prenant en compte l'apprentissage des étudiants, l'évaluation, le soutien et le suivi des apprentissages dans une approche par compétences. Généralement suivis en début de parcours, deux cours sont obligatoires : Modèles et stratégies d'enseignement au collège et à l'université (voir section 3.4pour le détail)et L'apprentissage au postsecondaire. Dans tous les cours, les activités en classe sont conçues en vue de développer chez les étudiants un changement conceptuel dans lequel les apprentissages sont transférés dans l'action concrète, observés, analysés, évalués et rajustés dans un processus de développement continue. Les travaux sont appliqués à la discipline de spécialisation de chaque étudiant et la réflexion s'appuie sur des problèmes complexes tirés de la réalité.

11 Depuis ses débuts, le cours sur les modèles d'enseignement, sur lequel s'appuie notre étude, aborde en 45 heures (15 séances de trois heures) l'apprentissage par problèmes (APP), la méthode des cas, l'apprentissage coopératif, la pédagogie par projets et l'exposé interactif. Le cours est construit de telle sorte que les étudiants (au nombre d'une vingtaine par groupe) vivent en classe les modèles d'enseignement à apprendre, c'est-à-dire que chacune des séances est enseignée par le biais du modèle à apprendre. De plus, les étudiants sont notamment évalués avec deux séances d'une heure qu'ils doivent planifier avec l'un des modèles vus en classe. Il importe de mentioner qu'aucun accompagnement pédagogique n'est effectué une fois la formation terminée.

\subsection{Les modèles d'enseignement enseignés au PCPES}

12 Les modèles d'enseignement enseignées au PCPES émanent de la recherche sur les pratiques enseignantes et sur l'apprentissage tout autant que d'un contexte de réformes en enseignement supérieur, particulièrement au niveau collégial. Sur le plan 
de la recherche, la littérature sur les modèles d'enseignement est abondante. Les recherches théoriques et empiriques sont multiples et abordent les modèles souvent séparément ou en fonction d'un contexte disciplinaire donné (Bell, 2010; Fayolle \& Verzat, 2009 ; Van Thien, Marguet, Favre \& Buty, 2013). Par exemple, nous retrouvons beaucoup d'écrits sur la méthode des cas dans les différents spécialités de l'administration (Crittenden, Crittenden \& Hawes, 1999; Ebrahimi \& Aktouf, 2005; Hertrich \& Mayrhofer, 2008; Marchesnay, 2010), ce qui apparaît logique puisque ce modèle a été développé dans les années 1920 par la Harvard Business School. Notons que certains modèles font l'objet de plus de recherches que d'autres. C'est le cas notamment de l'approche par problèmes et de l'apprentissage coopératif, où autant les apprentissages des étudiants que les façons de les utiliser ont été abondamment étudiés (voir à ce sujet Hung, Jonassen \& Liu, 2008, qui font une synthèse de près d'une centaine d'écrits sur l'APP ; Bédard, Lison, Dalle, Côté \& Boutin, 2012 ; Johnson \& Johnson, 2009). Plusieurs écrits témoignent d'expérimentations en classe de modèles d'enseignement et servent de guide pour les enseignants. Les ouvrages de Proulx (2008) et de Ménard et St-Pierre (2014) sont exemplaires à cet égard: ils exposent plusieurs expériences d'enseignement utilisant différents modèles. Parfois, les expérimentations sont implantées comme des approches programme et sont évaluées par les départements ou les facultés (Fayolle \& Verzat, 2009 ; Helle, Tynjälä \& Olkinuora, 2006). Parfois, ce sont des essais d'enseignants en classe qui publient leur expérience démontrant l'utilisation d'un modèle donné (Hmelo-Silver, 2004 ; Smith, Sheppard, Johnson \& Johnson, 2005).

13 Il n'est pas simple de répertorier les modèles d'enseignement à l'intérieur d'une classification claire. Comme il n'y a pas de consensus sur les nominations et les définitions des modèles d'enseignement, il règne une certaine confusion dans la littérature. À titre d'exemple, pour Raby et Viola (2007), l'apprentissage par problèmes (APP), l'apprentissage coopératif, la pédagogie par projets et la méthode des cas sont vus des modèles d'enseignement. Seul l'exposé interactif est considéré comme une méthode d'enseignement (cette classification est aussi celle du PCPES). Pour Legendre (2005) et Viennau (2005), l'apprentissage coopératif serait une stratégie d'enseignement, pour Raby et Viola (2007), c'est un modèle d'enseignement et pour Baudrit (2007), il s'agit une méthode pédagogique. Joyce, Weil et Calhoun (2004) ainsi que Raynal et Rieunier (2012) admettent qu'aucune classification n'est satisfaisante. Toutefois, les cinq grandes familles de modèles, qui en répertorient plus de quatrevingts, proposées par Joyce, Weil et Calhoun (2004) sont généralement acceptées par les les chercheurs dans le domaine.

14 Chez les enseignants du PCPES, un modèle d'enseignement est perçu comme un agencement d'activités pédagogiques et d'interventions, justifié par un cadre de références issu d'une représentation de l'apprentissage et validé empiriquement ou scientifiquement. Selon Raynal et Rieunier (2012), un «modèle d'enseignement est conçu pour tenter de développer chez les élèves des apprentissages particuliers et certaines dimensions de la personnalité humaine estimées essentielles par le modèle " (p. 329). Les modèles d'enseignement sont donc liés de près aux objectifs d'apprentissage à atteindre (Raby \& Viola, 2007). C'est l'une des raisons justifiant le choix des modèles à être enseignés au PCPES. Ils sont abordés non pas en déterminant la supériorité d'un modèle sur un autre, mais en fonction des objectifs d'apprentissage et du contenu à apprendre. Ainsi, si l'objectif est d'appliquer des notions, la méthode des cas est un choix judicieux. Les modèles d'enseignement enseignés au PCPES ont 
aussi été choisis parce qu'ils rendent les étudiants actifs (ils s'inscrivent donc dans les pédagogies actives) et dans l'optique de favoriser l'utilisation de modèles d'enseignement variés.

\subsection{Le cours du PCPES sur les modèles d'enseignement}

15 Pour offrir au lecteur un portrait plus explicite, nous détaillons ici le déroulement des séances au cours desquelles les modèles sont enseignés dans le cadre du cours « Modèles et stratégies d'enseignement au collège et à l'université » du PCPES.

\subsubsection{L'exposé interactif}

Dans le cadre du PCPES, l'exposé interactif correspond à un exposé magistral, privilégiant une conception transmissive encore très largement utilisée en enseignement supérieur, actualisé avec les principes issus des recherches sur l'apprentissage et en psychologie cognitive. Alors que l'exposé magistral est défini comme un exposé oral d'un professeur, expert du contenu (Legendre, 2005), l'exposé interactif vise à rendre les étudiants actifs dans ses apprentissages pour favoriser une rétention plus en profondeur (Vanpee, Godin \& Lebrun, 2008). La séance sur l'exposé interactif se donne évidemment par le biais d'un exposé interactif. Elle débute par une courte discussion sur leurs expériences avec des professeurs effectuant des exposés magistraux et le visionnement du film Teaching teaching and understanding understanding (2006). Puis, l'enseignant fait un exposé interactif soutenu par un diaporama qui régule l'exposé. On y retrouve plusieurs pauses d'apprentissage (activités de réflexion individuelle ou en petit groupe visant l'application, la compréhension ou la consolidation des contenus). La séance prend appui sur la recherche pour bien marquer l'importance de la planification, de l'interactivité constante, de rendre les étudiants actifs en leur proposant des courtes tâches de compréhension, d'application et d'analyse (Brauer, 2011; Kozanitis \& Chouinard, 2007; Langevin \& Bruneau, 2000 ; Svinicki \& McKeachie, 2011). Le contenu de cette séance expose ainsi à la fois l'utilité de l'exposé magistral (nécessaire) et la nécessité de le renouveler pour qu'il s'oriente davantage sur les apprentissages et sur un engagement soutenu des étudiants en classe.

\subsubsection{La méthode des cas}

17 Pour enseigner la méthode des cas au PCPES, nous avons choisi de suivre les trois phases prescrites par la méthode traditionnelle soit 1) l'analyse individuelle, 2) une discussion en petits groupes et 3) une séance plénière (Guilbert \& Ouellet, 2002 ; Ménard \& St-Pierre, 2014). À la suite de l'activation des connaissances antérieures sur la méthode des cas (qu'en savent les étudiants? L'ont-ils déjà vécue à titre d'étudiants?), la théorie sur ce modèle (sa provenance, ses fondements, son déroulement, etc.) est présentée par un exposé interactif. Les étudiants ont ensuite une heure pour résoudre, en équipe de cinq ou six, un cas calqué sur la réalité professionnelle. Ce dernier présente un problème tel que des enseignants de collège ont à affronter dans leurs pratiques et inclut des faits, des opinions et des éléments de l'environnement qui peuvent influencer les prises de décision. Le cas repose sur des concepts transférables et généralisables et la documentation nécessaire à sa résolution est fournie en classe par l'enseignant. Ce travail en équipe invite les étudiants à analyser, à comparer et à juger des arguments en vue d'en arriver à une conclusion et à 
une décision (Hermant, 2004; Hertrich \& Mayrhofer, 2008; Van Stappen, 1989). L'enseignant supervise les équipes et voit à l'avancement des solutions. Au cours de la dernière heure, chacune des équipes présente sa solution et une discussion suit sur l'apprentissage et l'utilisation d'une telle méthode.

\subsubsection{L’apprentissage coopératif}

L'apprentissage coopératif a pour principaux fondements l'interdépendance positive, la responsabilité individuelle, l'engagement actif et la facilitation sociale (Allport, 1920 ; De Koninck, 1996 ; Raby \& Viola, 2007). En vue de bien démontrer ces fondements aux étudiants du PCPES, la séance se déroule au moyen de la technique du casse-tête (Perkins \& Saris, 2001; William, 2004). Le texte d'Aylwin (1992) traitant des vingt principes d'une bonne stratégie pédagogique sert de prétexte pour faire vivre en classe un réel apprentissage coopératif. Pour maximiser le temps en classe, les étudiants doivent lire le texte avant la séance. L'enseignant forme à l'avance les équipes et distribue les rôles coopératifs. Les membres des équipes doivent s'approprier les principes d'Aylwin qui leur sont assignés et en faire une courte synthèse qu'ils exposeront aux autres équipes. Après quoi, ils doivent développer un plan de séance disciplinaire basé sur les principes d'Aylwin et le présenter devant le groupe. L'enseignant termine avec un exposé interactif sur l'apprentissage coopératif en faisant des liens avec les multiples études qui rapportent les effets positifs de ce modèle, comme le développement de l'autonomie, l'amélioration de la capacité de raisonnement et le degré d'engagement plus élevé (Howden \& Laurendeau, 2005; Landow, 1990 ; Raby \& Viola, 2007 ; Stevens \& Slavin, 1995).

\subsubsection{L'approche par problèmes (APP)}

La séance est donnée sous forme de micro-APP. Les étudiants sont regroupés en équipes de cinq ou six et lisent en classe la situation-problème, procèdent à l'identification des termes inconnus et du problème, formulent des hypothèses et se partage la recherche individuelle d'informations (Major, 2002; Ménard \& St-Pierre, 2014; Soucisse, Mauffette \& Kandlbinder, 2003). L'enseignant «joue le rôle de gestionnaire du processus d'analyse du problème plutôt que de pourvoyeur d'information ou de formules » (Bah, 2012, p. 197) et s'assure de ne donner aucune information quant au contenu à apprendre par l'intermédiaire de la situation-problème. Celle-ci sert de prétexte à l'apprentissage de nouvelles connaissances sur le micro-APP et aborde différents aspects de l'enseignement et de l'apprentissage dans une classe de collège. À la séance suivante, une plénière permet aux équipes de faire une mise en commun et une analyse des informations recueillies pour tenter de comprendre la situationproblème. Chaque équipe fait ensuite part de ses conclusions au groupe. La leçon se clôt avec une discussion sur les avantages et les inconvénients de l'APP, et particulièrement du micro-APP, pour les étudiants et les enseignants.

\subsubsection{La pédagogie par projets}

La pédagogie par projets (incluant ses variantes: apprentissage par projets, approche par projets, enseignement par projets) est présente dans les discours de formation depuis longtemps (Proulx, 2008). Dans le cours sur les modèles d'enseignement au PCPES et en accord avec Hubert (2005) pour qui « les connaissances sont construites par 
l'individu par l'intermédiaire des actions qu'il accomplit sur les objets »(p. 25), la pédagogie par projets est d'abord présentée avec une discussion sur les expériences vécues à titre d'étudiant. Le but ici est de faire ressortir les multiples projets possibles, de connaître le vécu des étudiants avec ce modèle et d'en identifier les avantages et les inconvénients. Ensuite, l'enseignant fait un exposé interactif sur la pédagogie par projets : son origine, ses applications, ses étapes, ses obstacles, etc. Les étudiants se placent par la suite en équipes disciplinaires de quatre ou cinq et élaborent un plan de séance faisant appel à la pédagogie par projets qui sera présenté en classe à la fin du cours. Cet exercice leur permet d'intégrer sur le champ les principes de la pédagogie par projets en utilisant leur créativité, en faisant appel à la coopération et en leur fournissant une perspective professionnelle aux enjeux mobilisateurs (Arpin \& Capra, 2000 ; Leduc, 2014).

21 Voilà donc rapidement situées les visées et les choix pédagogiques concernant les modèles d'enseignement au PCPES. De ce qui précède, il ressort que ces choix sont faits pour faciliter un réinvestissement des apprentissages dans un contexte d'approche par compétences. Les activités, les situations-problèmes, les lectures et autres contenus visent conséquemment à ce que les étudiants puissent mobiliser leurs ressources à bon escient et au bon moment lorsqu'ils enseigneront dans un collège. Pour les y préparer, les enseignants du PCPES insistent sur les raisons des actions. À travers le programme, plusieurs occasions sont offertes pour que les futurs enseignants ajustent leur enseignement aux réalités actuelles de leur discipline et pour qu'ils portent un regard critique sur le travail de l'enseignant. Nous visons à qu'ils puissent s'adapter aux multiples réalités de l'enseignement au collégial, mais aussi à ce qu'ils puissent euxmêmes favoriser en classe le transfert des apprentissages de leurs futurs étudiants (Tardif, 1999).

22 À la lumière de ce qui précède, toujours dans le but d'améliorer le PCPES, notre objectif de recherche était de vérifier dans quelle mesure et pour quelles raisons les diplômés du PCPES enseignant au collégial utilisent ou non les modèles d'enseignement appris en formation initiale.

\section{Une enquête pour mesurer le transfert vers les pratiques enseignantes}

\subsection{Les sujets}

Notre première collecte de données a été réalisée au cours des mois d'avril et de mai 2012. Considérant une situation particulière qui a touché le Québec durant pluieurs mois en 2012, nous avons effectué une seconde collecte de données au printemps 2013 pour augmenter notre échantillon. Ce dernier se compose au final des 102 diplômés du PCPES, entre 2006 et 2011, toutes disciplines confondues.

\subsection{L'instrumentation}

Pour atteindre nos objectifs, nous avons demandé aux participants de répondre à un questionnaire en ligne (sur LimeSurvey), comprenant des items à choix multiples et des items à réponses construites (voir annexe). Le questionnaire contenait quelques items généraux pour nous permettre d'obtenir des données sociodémographiques, sur leur 
statut actuel d'enseignant, sur le type de cours qu'ils donnent, etc. Bien que secondaires dans la présente étude, ces données nous offrent un portrait du cheminement de nos diplômés et de leur insertion professionnelle, utile pour nos étudiants actuels et pour le développement du programme. Ce questionnaire a été élaboré à partir des modèles d'enseignement enseignés au PCPES ainsi que de questionnaires existants sur divers diplômés dans le cadre d'évaluation de programme. Il a été construit principalement dans le but de savoir si les diplômés utilisent les modèles d'enseignement appris et d'identifier ce qui facilite ou ce qui restreint leur utilisation. Il a par ailleurs été validé par deux experts en pédagogie de l'enseignement supérieur.

\subsection{L'analyse des données}

Notre méthode d'analyse combine des données quantitatives (présentes en plus grand nombre) et des données de nature plus descriptive, toutes issues du questionnaire en ligne. Seules les réponses des 41 répondants enseignant dans un collège ont été retenues pour l'analyse (les autres n'ayant pas pu répondre à ces items puisqu'ils n'enseignent pas dans le réseau collégial). Les premières analyses (statistiques) nous ont permis d'identifier les modèles d'enseignement, appris au PCPES, selon la fréquence de leur utilisation en classe, selon le défi qu'ils représentent ou selon la préférence pour l'un ou l'autre de ces modèles. Les items indiquant ce qui facilite ou rend difficile l'utilisation de modèles variés ont aussi fait l'objet d'analyses statistiques, de même que ceux indiquant les encouragements du milieu professionnel à les utiliser. Pour les items descriptifs sur ce qui encourage ou restreint l'utilisation de modèles variés, des analyses de contenu ont été effectuées. Il faut noter également qu'il y a eu des données manquantes pour certains items, ce qui explique que les résultats présentés ci-dessous ne totalisent pas toujours 41 réponses.

\section{Les premiers résultats}

Les résultats issus de l'analyse de nos données démontrent que, parmi les 102 personnes qui ont répondu au questionnaire, 41 enseignent dans un collège et 5 enseignent ailleurs (les réponses de ces derniers n'ont pas été analysées parce que leur milieu d'enseignement est peu semblable au collégial). Les autres n'enseignent pas du tout et ou n'ont pas répondu aux questions portant sur leur situation professionnelle. Seulement 6 des 41 répondants qui enseignent dans un collège ont une permanence et la moyenne d'âge de nos répondants est de 35 ans. Ce dernier résultat nous apparaît logique puisque la majorité des étudiants au PCPES ont au moins un baccalauréat et plusieurs années de travail dans leur domaine. Notons que l'enseignement au collégial est souvent une réorientation de carrière à la suite de l'acquisition d'une expérience pratique dans une discipline.

Pour atteindre nos objectifs de recherche, le cœur de notre questionnaire portait sur les modèles d'enseignement utilisés. Nous pouvons dégager des tableaux 1 et 2 que l'exposé interactif arrive en tête avec 26 répondants qui disent l'utiliser souvent, 11 l'utilisent toujours. Les enseignants le préfèrent aussi aux autres modèles. L'apprentissage coopératif, la méthode des cas, l'approche par problèmes suivent avec une moyenne de 12 répondants qui affirment les utiliser à l'occasion ou souvent. Autour de 14 participants admettent ne jamais utiliser la méthode des cas et la 
pédagogie par projet et le quart n'utilisent jamais l'APP. L'exposé magistral semble être encore utilisé occasionnellement et 11 répondants le classe en $2^{\mathrm{e}}$ alors que 12 le place en dernière position quant au degré de préférence qu'ils lui accordent. Dégageons aussi que plus de la moitié accordent à la méthode des cas et à l'apprentissage coopératif un $3^{e}$ rang en terme de préférence. Ce dernier point nous apparait particulièrement intéressant puisque la méthode des cas émane des sciences administratives et fait souvent l'objet, dans les classes du PCPES, de résistance de la part des étudiants provenant d'autres domaines comme les sciences humaines ou artistiques.

Tableau 1. Les réponses relatives aux modèles d'enseignement utilisés

Modèles

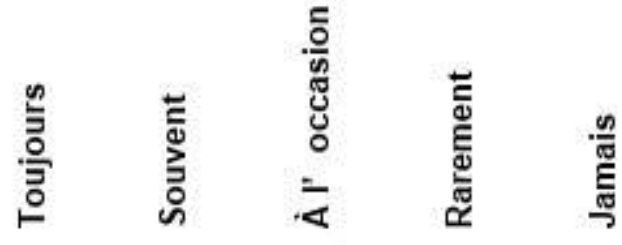

\begin{tabular}{r|ccccc|}
\cline { 2 - 6 } Exposé magistral & 3 & 9 & 11 & 10 & 8 \\
Exposé interactif & 11 & 26 & 4 & & \\
Cas & & 5 & 14 & 6 & 13 \\
Coop & 3 & 10 & 14 & 7 & 7 \\
APP & 1 & 2 & 15 & 10 & 10 \\
Projet & 2 & 9 & 8 & 4 & 14 \\
\cline { 2 - 6 } & & & &
\end{tabular}

Tableau 2. Les réponses relatives aux préférences

\begin{tabular}{|c|c|c|c|c|c|c|}
\hline Modèles & $1^{\text {er }}$ & $2^{\theta \mathrm{m} \theta}$ & $3^{\text {өme }}$ & $4^{\theta \mathrm{me} \theta}$ & $5^{\text {ตme }}$ & $6^{\mathrm{eme}}$ \\
\hline Exposé magistral & 2 & 11 & 6 & 6 & 4 & 12 \\
\hline Exposé interactif & 29 & 6 & 2 & 1 & 3 & \\
\hline Cas & & 6 & 15 & 5 & 6 & 8 \\
\hline Coop & 4 & 12 & 11 & 9 & 4 & 1 \\
\hline APP & 1 & 4 & 5 & 9 & 11 & 10 \\
\hline Projet & 5 & 2 & 2 & 10 & 12 & 9 \\
\hline
\end{tabular}

C'est donc dire que, parmi nos répondants, l'exposé interactif domine en ce qui concerne la fréquence et la préférence. L'exposé magistral est encore très présent, et ce, malgré la formation initiale dans laquelle nous insistons sur l'innovation pédagogique et les modèles variés. Toutefois, une précision s'impose : au PCPES, nous véhiculons aussi l'idée de renouveler l'exposé magistral traditionnel par le biais d'une plus grande interactivité avec les étudiants et d'une intégration constante de pauses 
d'apprentissage. Que l'exposé magistral soit le dernier choix en terme de préférence pour près du tiers de nos répondants est en soi une retombée positive.

Il semble que les modèles moins traditionnels, comme l'APP, soient laissés pour compte, particulièrement dans les domaines des sciences humaines, des arts et des lettres dont sont issus près de la moitié de nos répondants. Ce constat est tout de même étonnant puisque tous les programmes au niveau collégial sont offerts avec une approche par compétences, implantée graduellement entre 1993 et 2009. Ce tournant vers les compétences visait à renouveler la formation, à ajuster les programmes en fonction du marché du travail et à mettre en œuvre une véritable stratégie de réussite des études (Héon, Savard \& Hamel, 2008). Conséquemment, avec la mise en place de cette réforme, un changement de culture, ayant un impact sur toutes les tâches de l'enseignant dont celle de rendre les étudiants actifs, s'est imposé. En cohérence avec les particularités des compétences, nous pouvons supposer que les enseignants du collégial ont modifié leurs pratiques enseignantes pour rendre les étudiants actifs et responsables de leurs apprentissages (Bédard, 2006).

De plus, beaucoup d'efforts ont été mis dans le réseau collégial pour former les enseignants aux nouvelles réalités et pour les rendre prêts à accueillir les étudiants qui ont effectué tout leur cursus dans le cadre de la réforme en éducation, mise en place au primaire et au secondaire au début des années 2000. Il est encore plus étonnant de constater que, malgré ces changements importants dans le système scolaire québécois et les formations initiales en enseignement offertes, les pratiques enseignantes semblent ne pas avoir changé de manière significative. En effet, nos résultats indiquent (tableau 3) que la pédagogie par projets, la méthode des cas et l'APP représentent un défi d'application pour plus du quart des répondants, alors que l'exposé magistral et l'exposé interactif ne sont pas des défis pour les enseignants.

Tableau 3. Les réponses relatives au degré de défi ressenti par rapport aux modèles

\begin{tabular}{|c|c|c|c|c|c|c|}
\hline Modèles & $1^{\mathrm{er}}$ & $2^{\text {tme }}$ & $3^{\text {eme } \theta}$ & $4^{\mathrm{eme} \theta}$ & $5^{\text {tme }}$ & $6^{\ominus \mathrm{me} \theta}$ \\
\hline Exposé magistral & 2 & 1 & 3 & 4 & 11 & 20 \\
\hline Exposé interactif & 4 & 3 & 1 & & 20 & 13 \\
\hline Cas & 9 & 7 & 11 & 11 & 1 & 1 \\
\hline Coop & 4 & 8 & 15 & 11 & 1 & 2 \\
\hline APP & 7 & 12 & 8 & 8 & 2 & 3 \\
\hline Projet & 15 & 10 & 3 & 6 & 5 & 1 \\
\hline
\end{tabular}

Ceci expliquant cela, il est possible que nos répondants ne trouvent pas dans leur milieu d'enseignement les conditions adéquates pour utiliser couramment les modèles appris au cours de leur formation initiale. Le tableau 4 indique que l'exposé interactif est facilité par la plupart des conditions, que près du tiers de répondants les perçoivent comme étant facilitant pour la méthode des cas et l'approche par problèmes et que pour l'apprentissage coopératif, c'est l'expérience en enseignement, l'apprentissage des étudiants et le climat de travail qui semblent faciliter davantage son utilisation. Quant à la pédagogie par projets, c'est près de la moitié des répondants qui considèrent que le 
climat de travail, l'apprentissage des étudiants, l'expérience en enseignement et les réactions des étudiants face à la stratégie facilitent son utilisation. En somme, il semble que l'exposé interactif ne pose pas de problème pour une majorité de nos répondants et qu'entre le tiers et le quart des répondants voient les conditions énumérées plus haut (climat de travail, expérience en enseignement, etc.) comme facilitant les autres modèles (autre que l'exposé interactif).

Tableau 4. Les réponses par rapport aux conditions facilitantes

\begin{tabular}{r|ccccc} 
Conditions & Interactif & Cas & Coop & APP & \multicolumn{1}{c}{ Projet } \\
\cline { 2 - 6 } Environnement physique & 39 & 5 & 21 & 11 & 17 \\
Ressources disponibles & 37 & 6 & 17 & 12 & 16 \\
Taille groupe & 31 & 11 & 22 & 15 & 16 \\
Motivation étudiants & 26 & 14 & 17 & 16 & 15 \\
Autonomie étudiants & 29 & 10 & 20 & 14 & 17 \\
Gestion de classe & 31 & 15 & 21 & 14 & 17 \\
Apprentissage & 35 & 16 & 26 & 16 & 24 \\
Réactions face à la stratégie & 39 & 14 & 25 & 17 & 23 \\
Matière enseignée & 41 & 12 & 22 & 12 & 19 \\
Climat de travail & 39 & 19 & 27 & 21 & 25 \\
Expérience en enseignement & 38 & 15 & 26 & 19 & 24 \\
Gestion du temps & 38 & 10 & 15 & 10 & 12 \\
Planification & 39 & 13 & 23 & 13 & 17 \\
Évaluation & 37 & 13 & 21 & 15 & 16 \\
\cline { 2 - 6 } & & & & &
\end{tabular}

Quant à ce qui restreint l'utilisation des modèles d'enseignement (tableau 5), les résultats sont moins significatifs, les moyennes se situant autour du tiers des réponses. Il semble que plusieurs conditions nuisent à l'utilisation de tous les modèles à l'exception de l'exposé interactif. En revanche, ce dernier serait affecté par la motivation et l'autonomie des étudiants. Le climat de travail n'apparaît pas ici déterminant pour l'utilisation des modèles. 
Tableau 5. Les réponses par rapport freins et obstacles dans l'utilisation

\begin{tabular}{r|ccccc} 
Conditions & Interactif & Cas & Coop & APP & Projet \\
\cline { 2 - 6 } Environnement physique & 4 & 18 & 10 & 13 & 9 \\
Ressources disponibles & 3 & 17 & 11 & 11 & 12 \\
Taille groupe & 11 & 17 & 13 & 9 & 11 \\
Motivation étudiants & 16 & 12 & 14 & 9 & 11 \\
Autonomie étudiants & 14 & 18 & 15 & 12 & 13 \\
Gestion de classe & 9 & 12 & 11 & 12 & 10 \\
Apprentissage & 3 & 7 & 4 & 7 & 5 \\
Réactions face à la stratégie & 6 & 11 & 6 & 8 & 6 \\
Matière enseignée & 3 & 15 & 11 & 7 & 12 \\
Climat de travail & 1 & 4 & 3 & 1 & 7 \\
Expérience en enseignement & 2 & 12 & 6 & 9 & 4 \\
Gestion du temps & 4 & 18 & 16 & 17 & 17 \\
Planification & 5 & 15 & 9 & 15 & 13 \\
Évaluation & 6 & 11 & 10 & 9 & 12 \\
\cline { 2 - 6 } & & & & & 12 \\
\hline
\end{tabular}
de ces conditions et avec les données dont nous disposons, nous concluons qu'il n'apparaît pas facile pour les enseignants d'utiliser en classe divers modèles. Cependant, chacun d'eux demeurent tout de même présents dans leurs pratiques. D'ailleurs, près de la moitié de nos répondants disent être souvent encouragés par leur milieu à utiliser des modèles variés et une douzaine d'entre eux le sont occasionnellement en ce qui concerne le recours à la pédagogie par projets, l'apprentissage coopératif et l'APP. Seule l'utilisation de la méthode des cas est peu encouragée. Enfin, le questionnaire comportaient quelques questions ouvertes sur ce qui facilitent ou restreint l'utilisation de modèles variés. Selon nos répondants, l'utilisation de modèles variés maintient l'intérêt des étudiants, suscite une participation active et améliore l'apprentissage. En revanche, le manque de temps, la quantité de contenus à voir et le manque d'autonomie pour agir sur le contenu des cours sont des obstacles importants.

Il importe de garder à l'esprit le fait que les conditions d'insertion pour les nouveaux enseignants au collégial sont difficiles : ils ont souvent beaucoup de nouveaux cours à préparer, leur statut est précaire, ils doivent participer au développement des programmes, travailler efficacement en équipe, subissent des pressions de redditions de compte, etc. Les exigences sont nombreuses (St-Pierre, 2007). C'est sans compter les conceptions parfois très traditionnelles de l'enseignement qui se heurtent à des valeurs nouvelles présentes dans la société québécoise et dans les établissements, ne serait-ce qu'en ce qui concerne les approches par compétences. En terminant, il faut noter que les nouveaux enseignants ne bénéficient pas toujours d'accompagnement lors de leur insertion dans un collège. Pour ceux-là, une réelle mise en pratique des modèles d'enseignement appris au PCPES peut s'avérer plus difficile. 


\section{Conclusion}

Notre objectif de recherche visait à vérifier dans quelle mesure et pour quelles raisons des diplômés du PCPES qui enseignent au collégial utilisent les modèles d'enseignement appris. Nos résultats montrent que les exposés magistraux et interactifs semblent bien présents malgré les multiples innovations pédagogiques et malgré la formation adaptée au contexte actuel et soutenue par la recherche en pédagogie de l'enseignement supérieur. Cependant, les participants semblent aussi arriver à utiliser, dans une moindre mesure, les autres modèles appris.

Comme les conceptions jouent un rôle déterminant dans les pratiques enseignantes, les programmes de formation devraient mieux en tenir compte pour les faire évoluer. Il serait par exemple utile que les modèles d'enseignement soient mieux réinvestis transversalement, dans tous les cours du PCPES, de manière à opérer un changement conceptuel plus durable augmentant ainsi les possibilités de les utiliser en classe sur une base régulière.

Le milieu de travail semble aussi avoir un impact sur le choix des modèles d'enseignement par les enseignants. Sans accompagnement, le réinvestissement dans un contexte professionnel apparaît difficile à opérer (Bédard, 2006). Davantage de liens seraient donc à tisser entre les milieux de formation et les milieux de pratique, par exemple avec un accompagnement pédagogique lors de la première année d'embauche (ce que certains collèges offrent déjà, mais sans réel contact avec les formations initiales (St-Pierre, 2012). Il s'agit là d'un résultat important de l'étude sur le PCPES puisque cette option d'accompagnement pédagogique est déjà dans le plan de développement du programme.

En arrière-plan, notre étude avait aussi le désir de mieux connaître le cheminement de nos diplômés. Avec leurs réponses au questionnaire, même si ce sont des données uniques et auto-rapportées qui ne traduisent qu'une partie de la réalité (Kane, Sandretto \& Heath, 2002), nous pouvons certainement mieux suivre leur parcours et ajuster la formation du PCPES en conséquence. À ce propos, il faut souligner la volonté de certains enseignants à intégrer de nouvelles pratiques dans leur enseignement, en dépit de la précarité de leur situation, de la complexité de leurs tâches et des cultures locales qui sont parfois déterminantes pour les orientations pédagogiques. Bien qu'aucune formation initiale ne puisse vraiment pallier tous les obstacles, elles offrent tout de même une préparation adéquate au marché du travail en enseignant collégial : connaissance du milieu collégial et de l'éducation supérieure, capacité à bien planifier les cours, à s'adapter aux réalités complexes et prise de responsabilités dans les multiples rôles de l'enseignant. 


\section{BIBLIOGRAPHIE}

Allport, F.H. (1920). The influence of the group upon association and thought. Journal of experimental psychology, 3, 159-182.

Arpin, L. \& Capra, L. (2000). L'apprentissage par projets. Montréal : Chenelière/McGraw- Hill. Aylwin, U. (1992). Les principes d'une bonne stratégie pédagogique. Pédagogie collégiale, 5(4), 11-15.

Bah, M.B. (2012). Apports, limites et enjeux de l'apprentissage par problèmes pour le développement de compétences associées au champ de l'éducation relative à l'environnement : une étude de cas en milieu universitaire guinéen (Thèse de doctorat inédite). Université du Québec à Montréal.

Baudrit, A. (2007). L'apprentissage coopératif : origines et évolutions d'une méthode pédagogique. Bruxelles : De Boeck Supérieur.

Bédard, D. (2006). Enseigner autrement, oui mais pourquoi et comment ? Le cas d'un cours universitaire du premier cycle. Dans N. Rege Collet et M. Romainville (dir.), La pratique enseignante en mutation à l'université (pp. 83-101). Bruxelles : De Boeck.

Bédard, D., Lison, C., Dalle, D., Côté, D. J. \& Boutin, N. (2012). Problem-based and project-based learning in engineering and medicine: determinants of students' engagement and persistance. Interdisciplinary journal for problem-based learning, 6(2), 7-30.

Bell, S. (2010). Project-based learning for the $21^{\text {st }}$ Century: skills for the future. The clearing house: a journal of educational strategies, issues and ideas, 83(2), 39-43.

Bertrand, Y. (1998). Théories contemporaines de l'éducation. Montréal : Éditions Nouvelles.

Brauer, M. (2011). Enseigner à l'université : conseils pratiques, astuces, méthodes pédagogiques. Paris : Armand Colin.

Conseil supérieur de l'éducation (CSE) (2000). Réussir un projet d'études universitaires : des conditions à réunir. Québec : Ministère de l'éducation du Québec.

Crittenden, V.L., Crittenden, W.F. \& Hawes, J.M. (1999). The Facilitation and use of student teams in the case analysis process. Marketing education review, 9(3), 15-23.

Cullen, J., Richardson, S. \& O'Brien, R. (2004). Exploring the teaching potential of empiricallybased case studies. Accounting education, 13(2), 251-266.

De Koninck, G. (1996). À quand l'enseignement ? Plaidoyer pour la pédagogie. Montréal, Québec : Éditions logiques.

Ebrahimi, M. \& Aktouf, O. (2005). La méthode des cas en gestion : apprentissage ou cercle vicieux? Communication présentée lors de la $14^{\mathrm{e}}$ conférence internationale de management stratégique. Angers, France.

Fayolle, A. \& Verzat, C. (2009). Pédagogies actives et entrepreneuriat : quelle place dans nos enseignements? Revue de l'Entrepreneuriat 2(8), 1-15.

Feyfant, A. (2011). Effets des pratiques pédagogiques sur les apprentissages. Institut français de l'éducation, 65, 1-14.

Guilbert, L. \& Ouellet, L. (2002). Études de cas : apprentissage par problèmes. Sainte-Foy, Québec : Presses de l'Université du Québec. 
Helle, L., Tynjälä, P. \& Olkinuora, E. (2006). Project-based learning in post-secondary education: theory, practice and rubber sling shots. Higher Education, 51(2), 287-314.

Héon, L., Savard, D. \& Hamel, T. (2008). Les cégeps : une grande aventure collective québécoise. SainteFoy, Québec : Presses de l'Université Laval.

Hmelo-Silver, C.E. (2004). Problem-based learning: what and how do students learn? Educational psychology review, 16(3), 235-266.

Hermant, J. (2004). La méthode des cas en gestion adaptée aux différents environnements socioculturels. Actes du colloque de la Cidegef. Beyrouth, Liban : Université Saint Joseph, Conférence internationale des dirigeants des institutions d'enseignement supérieur et de recherche de gestion d'expression française.

Hertrich, S. \& Mayrhofer, U. (2008). Cas en marketing. Cormelles-le-Royal, France : Editions management et société.

Howden, J. \& Laurendeau, F. (2005). La coopération : un jeu d'enfant. Montréal : Chenelière Éducation.

Hubert, M. (2005). Conduire un projet-élèves. Paris : Hachette.

Hung, W., Jonassen, D.H. \& Liu, R. (2008). Problem-based learning.Dans D.H. Jonassen (dir.), Handbook of research on educational communications and technology (pp. 485-506), Mahwah, NJ: Lawrence ErlBaum Associates.

Johnson, D.W. \& Johnson, R.T. (2009). An educational psychology success story: social interdependence theory and cooperative learning. Educational researcher, 38(5), 365-379.

Joyce, B., Weil, M. \& Calhoun, E. (2004). Models of teaching. Boston, MA: Allyn and Bacon.

Kane, R., Sandretto, S. \& Heath, C. (2002). Telling half the story: A critical review of research on the teaching beliefs and practices of university academics. Review of Educational Research, 72(2), 177-228.

Kozanitis, A. \& Chouinard, R. (2007). Une analyse exploratoire d'un modèle prédictif de la participation verbale en classe universitaire. Revue des sciences de l'éducation, 34(3), 711-728.

Langevin, L. (2007). Formation et soutien à l'enseignement universitaire : des constats et des exemples pour inspirer l'action. Sainte-Foy, Québec : Presses de l'Université du Québec.

Langevin, L. \& Bruneau, M. (2000). Enseignement supérieur : vers un nouveau scénario. Issy-lesMoulineaux: ESF éditeur.

Landow, G. (1990). Hypertext and collaborative work: the example of intermedia. Dans J. Galegher, R. Kraut \& C. Egido (dir.). Intellectual teamwork: social and technological of cooperative work (pp. 407-428). New York : Lawrence Erlbaum Associates.

Leduc, D. (2014). La pédagogie par projets. Dans L. Ménard \& L. St-Pierre (dir.), Se former à la pédagogie de l'enseignement supérieur (pp. 131-155). Montréal : Chenelière Éducation.

Loiola, F. \& Tardif, M. (2001). Formation pédagogique des professeurs d'université et conceptions de l'enseignement. Revue des sciences de l'éducation, 27(2), 305-326.

Legendre, R. (2005). Dictionnaire de l'éducation. Montréal: Guérin.

Major, C.H. (2002). Problem-based learning in general education at stanford university: a case study of changing faculty culture through targeted improvement efforts. The journal of general education, 51(4), 235-256. 
Marchesnay, M. (2010). La méthode des cas : pour un retour aux sources. Revue des cas en gestion, 2 , 5-22.

Ménard, L. (2009). Du cégep au baccalauréat : diversification des parcours et des expériences. Revue des sciences de l'éducation, 35(1), 169-190.

Ménard, L. \& St-Pierre, L. (2014). Se former à la pédagogie de l'enseignement supérieur. Montréal : Chenelière éducation.

Meirieu, P. (2009). L'école, mode d'emploi : Des « méthodes actives » à la pédagogie différenciée. Issy-lesMoulineaux : ESF éditeur.

Perkins, D.V. \& Saris, R.N. (2001). A jigsaw classroom technique for undergraduate statistics courses. Teaching psychology, 28(2), 111-113.

Proulx, J. (2008). L'apprentissage par projet. Sainte-Foy, Québec : Presses de l'Université du Québec.

Raby, C. \& Viola, S. (2007). Modèles d'enseignement et théories d'apprentissage : de la pratique à la théorie. Anjou, Québec: CEC.

Ramsden, P. (2003). Learning to teach in higher education. London: Routledge and Falmer.

Raynal, F. \& Rieunier, A. (2012). Pédagogie, dictionnaire des concepts clés. Apprentissage, formation, psychologie cognitive. Issy-les-Moulineaux : ESF éditeur.

Romainville, M. (2004). Esquisse d'une didactique universitaire. Prix des innovations pédagogiques. Conférence internationale des dirigeants des institutions d'enseignement supérieur et de recherche de gestion d'expression française et Agence Universitaire de la Francophonie, Paris, France.

Smith, K.A., Sheppard, S.D., Johnson, D.W. \& Johnson, R.T. (2005). Pedagogies of engagement: classroom-based practices. Journal of engineering education, 94(1), 87-101.

Soucisse, A., Mauffette, Y. \& Kandlbinder, P. (2003). Les problèmes : pivots de l'apprentissage par problème (APP) et de la motivation. Res Academica, 21(1), 129-150.

St-Pierre, L. (2007). Enseigner au collégial aujourd'hui. Pédagogie collégiale, 20(2), 5-12.

St-Pierre, L. (2012). La formation continue et l'accompagnement du personnel enseignant du collégial. Leçons tirées de l'innovation et de la recherche. Montréal : Chenelière Éducation.

St-Pierre, L., Bédard, L. \& Lefebvre, N. (2012). Enseigner dans un programme universitaire innovant : de nouveaux rôles à apprivoiser, des actes pédagogiques à diversifier. The canadian journal for the scholarship of teaching and learning, 3(1), 1-24.

Stevens, R. \& Slavin, R. (1995). The cooperative elementary school: effects on student's achievement, attitudes, and social relations. American educational research journal, 32(2), 321-351.

Svinicki, M. \& McKeachie, W. J. (2011). McKeachie's teaching tips: strategies, research and theory for college and university teachers.Belmont, CA : Wadsworth, Cengage Learning.

Tardif, J. (1999). Le transfert des apprentissages. Montréal : Éditions Logiques.

Vanpee, D., Godin, V. \& Lebrun, M. (2008). Améliorer l'enseignement en grands groupes à la lumière de quelques principes de pédagogie active. Pédagogie médicale, 9, 32-41.

Van Stappen, Y. (1989). L'enseignement par la méthode des cas : nature et fonction, techniques d'application, types d'apprentissage. Joliette, Québec : Cégep de Joliette-De Lanaudière.

Van Thien, N., Marguet, C., Favre, B. \& Buty, C. (2013). Pédagogie par projet : une étude de cas dans l'enseignement supérieur technologique. Revista Ensaio Belo Horizonte, 15(1), 15-30. 
Viennau, R. (2005). Apprentissage et enseignement : théories et pratiques. Montréal, Québec: Gaëtan Morin/Chenelière Éducation.

William, D. (2004). Improving race relations in higher education: the jigsaw classroom as a missing piece to the puzzle. Urban education, 39(3), 316-344.

\section{RÉSUMÉS}

$\mathrm{Au}$ Québec, nombreux sont les collèges qui valorisent une formation initiale en enseignement supérieur au moment de l'embauche de leurs enseignants. Pour répondre à cette exigence, l'Université du Québec à Montréal a développé une formation en pédagogie de l'enseignement supérieur (PCPES). Dans le cadre de cette formation, plusieurs modèles d'enseignement sont enseignés. Ces derniers auraient un impact sur les apprentissages réalisés par les étudiants et ils ne peuvent être dissociées des contextes dans lesquels ils prennent place. En effet, plusieurs éléments propres au milieu d'accueil favorisent ou restreignent les décisions pédagogiques et l'utilisation de modèles d'enseignement variés. C'est pour mieux arrimer la formation initiale aux pratiques en salles de classe que les diplômés du PCPES, qui ont obtenu une charge d'enseignement dans un collège, ont répondu à un questionnaire portant sur les modèles d'enseignement qu'ils utilisent au quotidien. Avec cette modeste étude, nous visions à décrire ce qui facilite ou restreint l'utilisation des modèles d'enseignement en salle de classe chez de nouveaux enseignants au collégial diplômés du PCPES. Est-ce qu'ils utilisent les modèles appris au cours de leur formation initiale? Une partie d'entre eux seulement ont l'occasion de les mettre en pratique et, pour une majorité d'entre eux, l'exposé interactif domine leur enseignement.

Cette recherche a été réalisée avec le soutien financier du Programme facultaire d'aide financière à la recherche et à la création (PAFARC) pour les nouveaux chercheurs de l'Université du Québec à Montréal.

In Quebec, there are many colleges that promote initial training in higher education when hiring teachers. To meet this requirement, the University of Quebec in Montreal has developed an initial training in teaching in higher education (PCPES). As part of this training, several teaching models are taught. Teaching approaches have an impact on the learning achieved by students and they cannot be separated from the contexts in which they take place. Indeed, several host environment elements facilitate or forces pedagogical choices and the use of various teaching models. So as to align initial training and teaching practices in classrooms, graduates of our PCPES, who have obtained a teaching job in a college, answered an online questionnaire on the teaching approaches they use every day. With this study, we aim to describe what facilitates or constrains the use of teaching models in the classroom by beginner college teachers who received initial training in pedagogy. Do they use the approaches taught during their initial training? Only a few of them have the opportunity to put into practice the approaches, and for a majority of them, the interactive lecture still dominates their teaching.

\section{INDEX}

Mots-clés : collégial, formation des enseignants, modèles d'enseignement, pédagogies actives, pratiques enseignantes 


\section{AUTEURS}

\section{DIANE LEDUC}

Département de didactique, Université du Québec à Montréal, Montréal, Canada leduc.diane@uqam.ca

\section{LOUISE MÉNARD}

Département de didactique, Université du Québec à Montréal, Montréal, Canada menard.louise@uqam.ca

\section{ÉRIC LE COGUIEC}

Département de didactique, Université du Québec à Montréal, Montréal, Canada

le_coguiec.eric@uqam.ca 